\title{
Optical flow and viewpoint change modulate the perception and memorization of complex motion
}

\author{
MOHAMED JARRAYA and MICHEL-ANGE AMORIM \\ Université de Paris-Sud 11, Orsay, France \\ and \\ BENOÎT G. BARDY \\ Université de Paris-Sud 11 and Institut Universitaire de France, Orsay, France
}

\begin{abstract}
Participants observed a point-light character (PLC) performing a gymnastic movement. They either memorized the final PLC orientation from the initial viewpoint, to match it to a test posture (memory task), or judged whether the biological motion appeared continuous (perceptual task), despite a viewpoint change. The observer could be either static or virtually in motion (pan or track) while looking at the movement from the initial viewpoint. The presence of a spatial layout during virtual self-motion induced a global optical flow specifying the translational component of the PLC movement, rendering the event more predictable for the participants. A representational momentum effect was observed in the memory task, suggesting that when a visual stimulation, such as a PLC motion, is abruptly stopped, its dynamics survive. In contrast, structural and transformational invariants specifying the PLC motion were sufficient to solve the perceptual task accurately. Finally, both the remembering of the final posture and the perception of continuity degraded with an increase in viewpoint change due to tilt/slant posture orientation matching, indicating that orientation processes interfered with event perception.
\end{abstract}

There has been vigorous debate regarding the theoretical model that best explains the nature of event perception. For the proponents of top-down theories (indirect perception), in order to perceive an event, the succession of stimuli must be strung back together to reconstitute the dynamic event. For the proponents of the theory of direct perception, the information is not partitioned, specifies the event, and therefore, need only be detected, not mentally reconstituted (Michaels \& Carello, 1981). The perception of biological movement is a particularly interesting case of event perception. How we perceive biological motion despite the impoverishment of the stimulus has been at the heart of the study of motion perception these last three decades. It is a well-known fact that the visual perception of events involving a biological creature can be supported by purely kinematic patterns (Runeson \& Frykholm, 1983). Johansson's (1973) point-light displays have been used extensively to show that gender, identity, amount of weight being lifted, and emotional expressions can be retrieved from kinematic patterns (transformational invariants), as well as from geometric, informationally relevant patterns (structural invariants; see,

The research reported in this article was supported by Enactive Interfaces, a network of excellence (IST Contract 002114) of the Commission of the European Community, and by Grant BQR-RV-2003 from the University of Paris Sud-11. Correspondence concerning this article should addressed to M. Jarraya, Research Center in Sport Sciences, University of Paris Sud-11, 91405 Orsay Cedex, France (e-mail: mohamed.jarraya@ staps.u-psud.fr). e.g., Dittrich, 1993; Johansson, 1973; Thornton, Pinto, $\&$ Shiffrar, 1998; Verfaillie, 2000). In this article, we will examine a specific aspect of event perception: its anticipatory component.

Anticipation is one major component subserving precise event perception, as is revealed by many examples in sports or in everyday life. For instance, our knowledge of biomechanical constraints (Shiffrar \& Freyd, 1990) can be used to anticipate forthcoming sequences of biological movements. Similarly, experts are better than novices at using advance visual information for anticipating future actions (Abernethy, 1987; Ste-Marie, 2000; Williams, Davids, Burwitz, \& Williams, 1994) and facilitating decision making (Alain \& Proteau, 1980). Interesting for the present article is, however, the reverse finding that anticipation can lead to misjudgments. Likewise, a witness observing a car accident might wrongly attribute the fall of a pedestrian to the arrival of a car, when in fact, there had been no collision. This misjudgment is consistent with Yela's (1952) earlier work reporting that perception of causality is observed despite a spatial gap between the approaching launcher and the beginning of the launched target motion. Here, we assume that some of these misjudgments reflect the anticipatory function of our visual system. Representational momentum (RM) effects (the offset position of a moving target is mislocalized in the direction of a movement) and boundary extension effects (the remembered view of a scene expands to include a region just outside the boundaries of the original view) are well-documented illustrations of the adaptive value of predictability in the visual 
system (see Thornton \& Hubbard, 2002, for a review). Hubbard and Bharucha (1988) suggested that the RM effect, observed for visually moving stimuli, reflects the anticipated, rather than the actual, direction of target motion. This effect occurs because there is a natural tendency to mentally extrapolate the motion of a target stimulus into the future. This anticipatory effect reflects our incapacity to stop the extrapolation of a moving target exactly at its disappearance (Finke, Freyd, \& Shyi, 1986; Freyd, 1987). In this article, we will concentrate on the RM effect and will provide evidence, using realistic sports-related biological movements, that RM can be considered as a variable reflecting the anticipatory function of the visual system.

\section{Optical Flow and Representational Momentum}

In everyday life, optical flow is useful for specifying the characteristics of the perceived event. Humans and other animals are often moving in an environment in which elements (objects and creatures) are themselves in motion. It is well established that the displacement of the point of observation in a stationary environment (selfmotion) produces a global transformation in the structure of the optic array, called global optic flow, and that an object (or a person) moving relative to a stationary observer produces a local transformation in a bounded region of the optic array, called local optic flow (Gibson, 1950; Warren, 1995). Hochberg (1986) nicely reported that filmmakers use very different visual flow techniques, such as the pan or the track shot, in order to simulate the same information about the whole scene.

Figure 1 illustrates the position and movement of a virtual observer (i.e., the camera) in three conditions of observation-(A) static, (B) pan, and (C) track-of a point-light character (PLC) performing an acrobatic movement (a round-off/backward-somersault). These three conditions were used in our study. In both the static and the pan conditions, the camera remains in the same position; however, in the pan condition, the camera is centered on the PLC and rotates around the vertical axis in order to keep the PLC in the center of the field of view. If we assume that the virtual observer's gaze is anchored on the PLC in the static condition, these two conditions roughly produce the same retinal flow. ${ }^{1}$ In addition, because the distance between the observer and the PLC changes similarly during the movement, the two conditions produce the same local optical flow. In the track condition, the virtual observer is translating along a line parallel to the PLC displacement, with a constant observer-PLC distance, thus producing (local) optical flow patterns different from those for the static and the pan conditions. Finally, in terms of global optical flow, the static condition produces no global flow pattern at the virtual observation point; the track condition produces a global translational flow pattern due to lateral self-motion; and the pan condition produces a global rotational flow pattern due to head rotation, with magnitudes of flow velocity vectors independent of element distances (Warren, Mestre,

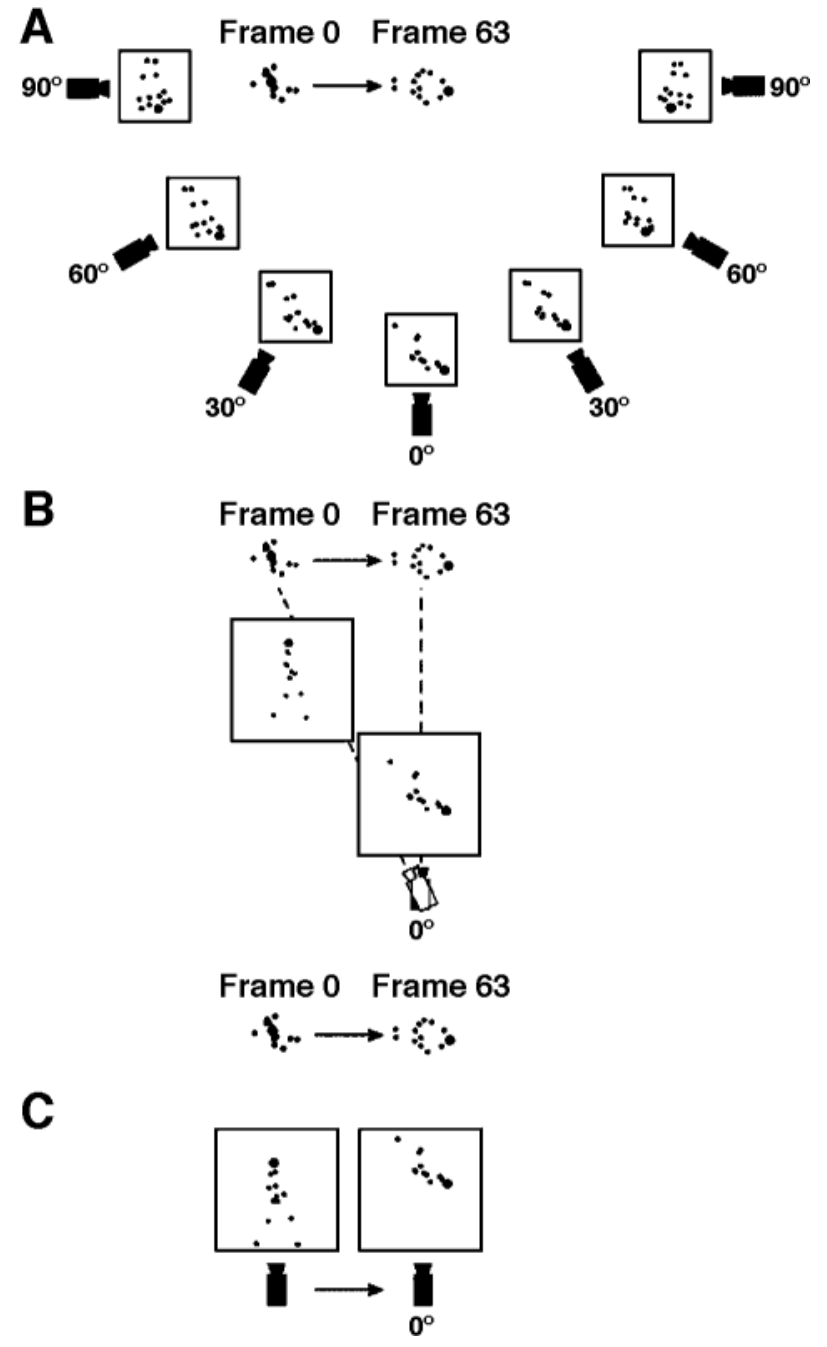

Figure 1. Map view of camera configurations and local views on a point-light character (PLC) performing a round-off/backwardsomersault in three conditions of observation (static, pan, and track). (A) The local views at Frame 63 (corresponding to a particular posture within the somersault) from different static cameras. (B) The pan camera is centered on the PLC and rotates about the vertical axis. (C) The track camera is translating with the moving character. The line of sight of the track camera is always perpendicular to the PLC trajectory.

Blackwell, \& Morris, 1991). ${ }^{2}$ Hence, the global optical flow structure differs between the pan and the track conditions. However, as the distance between the observer and the PLC increases, the lamellar components of the two flow patterns become increasingly similar, reducing the difference between the two flow structures.

Following the laws of ecological optics (Gibson, 1958), the analysis above suggests that the presence and structure of optical flow for an observer evaluating the movement of another observer (such as the PLC) can facilitate the evaluation of that movement. In this study, we expected that in the absence of optic flow specifying the translational or rotational component of the PLC movement, 
the event would be less predictable and anticipatory effects, such as the RM effect, would not occur. This phenomenon, or the reverse phenomenon that RM increases with the enrichment of the flow structure, has been occasionally reported but never tested systematically. Thornton and Hayes (2004) have recently reported that the forward shifts classically associated with RM could be observed with complex dynamic displays depicting human action (such as short video sequences of complex crowd scenes: a high school entrance, a town square, a department store, and a railway station). Similarly, Verfaillie and Daems (2002) have indicated that judging whether a static posture shows a possible or impossible body configuration is facilitated by the previous presentation of a motion sequence that would have resulted in the posture if the human action had continued. Finally, Whitney and Cavanagh (2002) have shown that RM is influenced by the direction and velocity of surrounding motion. In Experiment 1, the translational component of a simulated round-off/backward-somersault movement was impoverished (1) by using camera motions such as a pan (the viewer's rotation about the vertical axis) or a track (the viewer's translation perpendicular to the line of sight) and/or (2) by eliminating background cues producing optical flow during a pan and a track. As a consequence, if the moving character were to disappear abruptly, we would predict that representational momentum effects should be reduced in these conditions.

\section{The Perception of Continuity Across Viewpoint Change}

In an attempt to generalize the findings of the first experiment concerning the modulation by observation con- ditions of top-down anticipatory effects, in a second experiment, we designed a perceptual task, the detection of motion continuity, that could be performed without a high level of cognitive inference. We indeed expected that this task would not require the memory processes that are subject to RM effects. In Experiment 2, we predicted that structural and transformational invariants specifying a biological motion would be sufficient to accurately judge motion continuity across viewpoint changes (camera cuts). More precisely, we predicted that the amplitude of the viewpoint change, rather than the conditions of observation per se (whether the virtual observer was static or in motion relative to the moving character), would contribute to the perceived event continuity. This prediction was based on empirical filmmaking rules, such as directional continuity across cuts, that are used to facilitate an observer's spatial orientation and his/her locus of attention across views (d'Ydewalle \& Vanderbeeken, 1990). According to this rule, successive shots should preserve the participant's direction of movement across cuts (Arijon, 1993). In Experiment 2, we restricted our manipulation to viewpoint changes introduced either along or on the same side as the line of action - that is, the direction of the biological motion. As a consequence, the viewpoint change effect we observed on perceived event continuity would be due to the change from a tilt to a (more ambiguous) slant rotation percept with increasing difference in viewpoint.

\section{EXPERIMENT 1}

In this experiment, participants observed a threedimensional (3-D) PLC performing a gymnastic move-

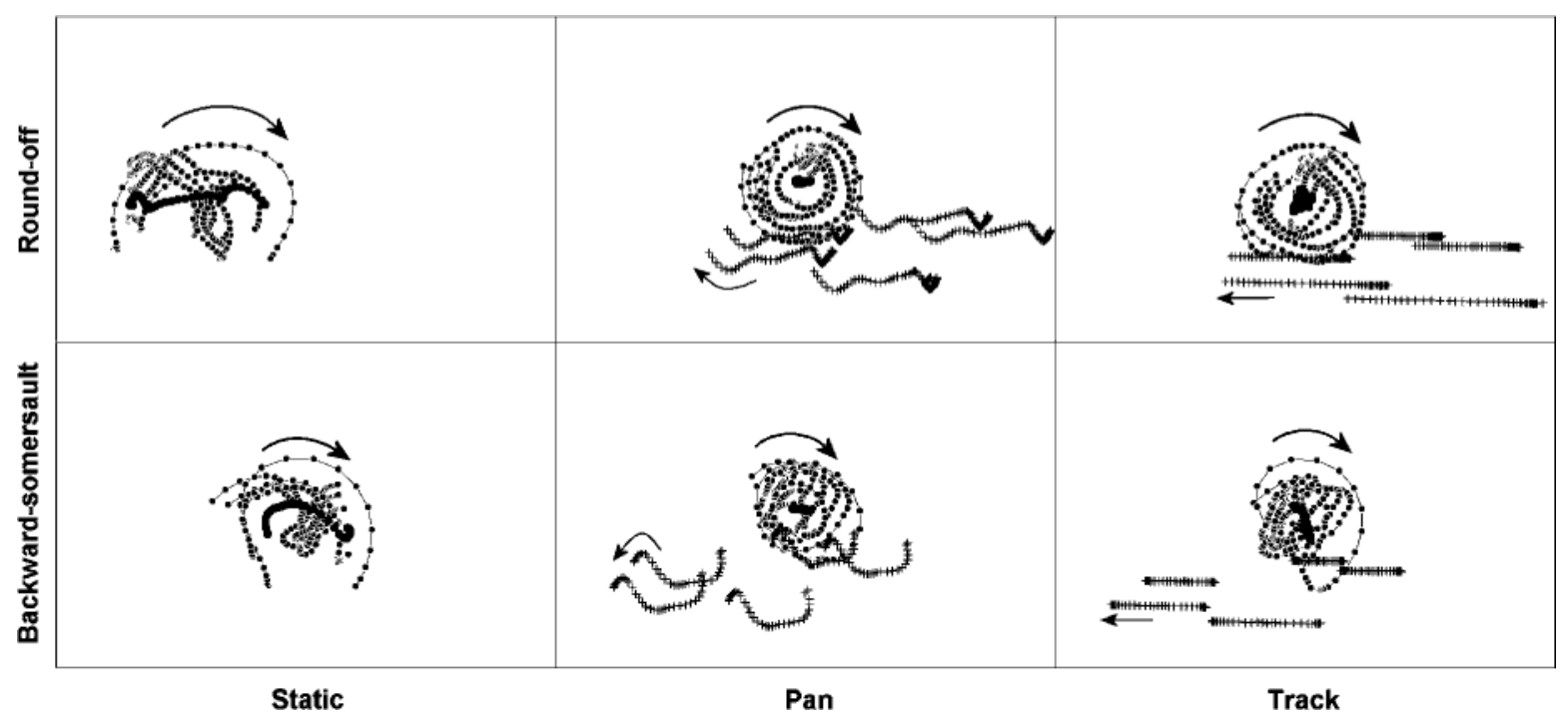

Figure 2. Trajectory of the point-light character (PLC) left body side (head, shoulder, elbow, wrist, hip, knee, and ankle) in the static, pan, and track camera conditions during execution of the round-off (from Frame 0 to Frame 50)/backward-somersault (from Frame 51 to Frame 92). The trajectory of the hips is indicated with thicker dots. In addition to the PLC trajectory, the visual flow due to the motion of the camera (crosses), relative to the spatial layout, is indicated for pan (rotational) and track (translational) cameras. The arrows indicate the direction of the PLC and spatial layout movements. As is evidenced, pan and track conditions engender very similar flow patterns. 
A

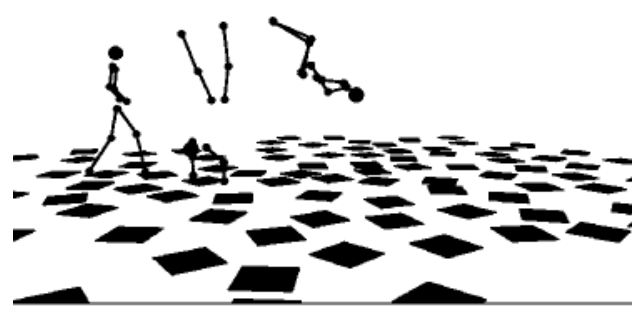

B
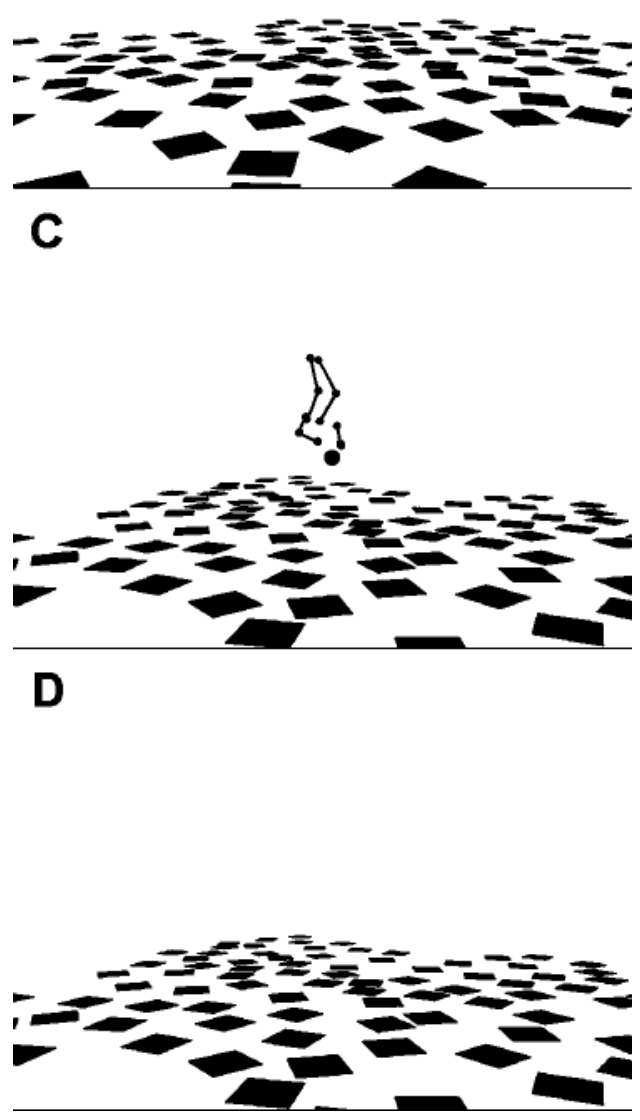

Figure 3. Example of the displays in the spatial layout condition for a static observer (Experiment 1). The somersault sequence (A; here, Frames 0,40 , and 63, are superimposed) was followed by an empty view $(B ; 1,500 \mathrm{msec})$ and by a brief presentation $(500$ msec) of a test posture (Frame 64) (C and D). The observer's viewpoint changed between $B$ and $C\left(\right.$ here,$\left.+60^{\circ}\right)$. In order to facilitate the reader's comprehension of the movement of the PLC, the spheres representing the joints are connected with lines in this figure that were not present in the animated sequences presented to the participants. ment — namely, a round-off/backward-somersault, interrupted during the backward somersault. They were asked to remember the final posture at the time of interruption and to compare it with a test posture presented later on. While memorizing the final posture, the observers could be either static or in motion (pan or track), and a change in the viewpoint was introduced, from $0^{\circ}$ to $90^{\circ}$ between the final and the test postures (see Figure 1). The central question was whether global optical flow would modulate the memory of the final posture of the perceived PLC. We expected that in the absence of optic flow specifying the translational component of the PLC movement, the event would be less predictable and anticipatory RM effects would not occur.

\section{Method}

Participants. Nineteen individuals, whose ages ranged from 23 to 41 years, participated in this experiment. All had normal or corrected-to-normal vision and were naive with respect to the hypotheses under investigation.

Apparatus and Stimuli. The stimuli were displayed on and the data collected by a PC using ERTS-IPL, a PC-compatible software package that allows development and performance of psychological experiments (Beringer, 1994). The participants viewed the display on a 21-in. monitor from a distance of $57 \mathrm{~cm}$. The experiment was conducted in a dimly lit room. The animations of the 3-D PLC stimuli were created using 3D Studio MAX, on the basis of an offthe-shelf example from Character Studio. The PLC was composed of 13 spheres corresponding to the head (1), shoulders (2), elbows (2), wrists (2), hips (2), knees (2), and ankles (2), as illustrated in Figures 2 and 3.

Procedure. In order to make sure that the observers recognized the movement of the PLC, before the beginning of the experiment, they were randomly presented the whole round-off/backwardsomersault (27 frames/sec) in the different viewing conditions under study (cf. Figures 1 and 2). In the experimental trials, the participants had to memorize the final posture of the PLC, which then disappeared during the backward somersault for $1,500 \mathrm{msec}$, before reappearing briefly $(500 \mathrm{msec})$ in a static posture, in order to decide whether the latter test posture was located after or before the actual final posture, with respect to the direction of motion. A typical experimental trial is illustrated in Figure 3. The participants initiated the trials by pressing the space bar on the keyboard and answered using the Ctrl keys (before = left key; after = right key). They were instructed to respond as quickly and accurately as possible. If reaction time (RT) to the test posture onset exceeded $6 \mathrm{sec}$, a "Wake up!" message was presented on screen. The posture test corresponded to frames $n-2, n-1, n, n+1$, and $n+2$, given that the frame of the posture to be memorized was frame $n$. The virtual observer was the camera that had been used to generate the visual scene, whereas the real observer was the experimental participant. Depending on the viewing condition, the virtual observer could be either static or in motion (pan, rotation of the viewpoint about the vertical axis; track, translation of the observer with the moving character) while observing the PLC movement. The static, pan, and track conditions all generated a local optical flow, due to the translation and/or rotation of the PLC relative to the observer. The pan and the track conditions produced different global flow patterns at the virtual observation point (rotational and translational, respectively), and the static condition added a visual flow component for the real observer in screen coordinates, due to the translation of the PLC relative to the screen (Figure 1). Flow patterns were created by displaying a fixed spatial layout in addition to 


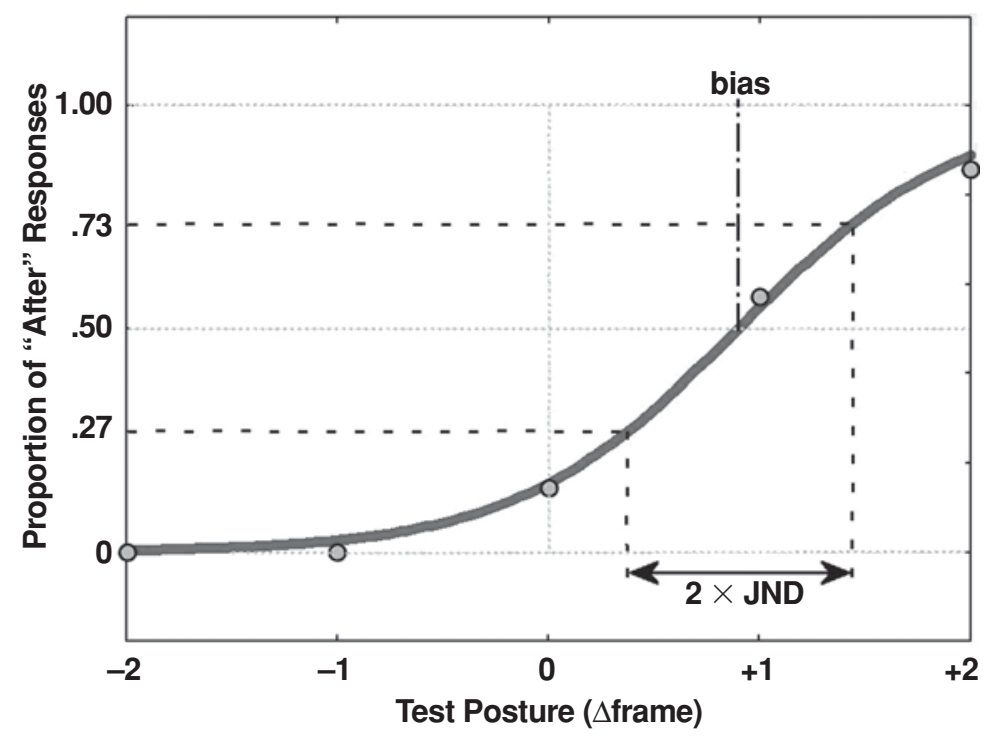

Figure 4. The point of subjective equality (PSE) and the just noticeable difference (JND) provide two independent measures. The PSE (bias) reflects the accuracy of the responses. In contrast, the JND evaluates the precision of the remembered orientation. In this example, Subject 15 has a bias of 0.90 and JND value of 0.53 in the track condition in the presence of the layout.

the PLC (Figure 2) and were suppressed by removing the layout. Finally, a change in viewpoint was introduced between the final and the test postures, varying between $0^{\circ}$ and $90^{\circ}$ (Figures 1 and 3 ).

The experimental session began with 12 practice trials, not included in the data set. Both the practice and the experimental trials were presented in a different random order for each participant and without feedback. Each participant performed 210 experimental trials: 2 layout conditions (with vs. without spatial layout) $\times 3$ conditions of observation (static vs. pan vs. track) $\times 7$ angular differences $\left(-90^{\circ},-60^{\circ},-30^{\circ}, 0^{\circ},+30^{\circ},+60^{\circ}\right.$, or $\left.+90^{\circ}\right) \times 5$ test postures $(n-2, n-1, n, n+1$, or $n+2)$. The final posture to be memorized could be that of Frame 63, 66, or 69. These final postures were crossed with the other factors in an incomplete balanced design (Cochran \& Cox, 1957).

Dependent variables. Points of subjective equality (PSEs) were computed by fitting sigmoid curves to the responses of each participant at the various test postures, using a logistic function, in order to infer the final posture memorized. The test posture was the same as either the final displayed posture or that in the one or two frames before or after. PSE values corresponded to the memory bias reflecting the accuracy of the responses (Figure 4). A value of PSE equal to 0 indicated that the participant's performance was accurate. A memory bias equal to 1 indicated that the memorized final posture was shifted in the direction of the movement, about one frame from the actual final posture. Such a positive memory bias reflects an RM effect. Note that each frame being presented for $37 \mathrm{msec}$, the bias values can be converted in time. A bias equal to one frame corresponds to a 37-msec memory bias. However, for the sake of simplicity, we will present the results in units of frames $(1$ frame $=$ $37 \mathrm{msec}$ ), rather than in milliseconds.

Just noticeable difference (JND) values were computed, in order to evaluate the precision of the remembered orientation (Figure 4). JND is the minimum amount by which a stimulus must be changed to produce a noticeable difference. It is the difference between two stimuli (actual vs. remembered final posture) that is detected as often as it is undetected. In other words, the greater the JND values, the shallower the sigmoid slope (see Figure 4), and the less precise the remembered posture. It should be noted that PSE and JND provide two independent and complementary measures of performance. Finally, RTs (in milliseconds) to the test posture were also recorded.

\section{Results and Discussion}

Across the two experiments, the significance level was fixed to $p<.05$ (but for Bonferroni corrections); however, $p$ values for marginally significant $(p<.08)$ results will be reported.

Conditions of observation. The first point to note is that the average memory bias in Experiment 1 was positive $(M=0.36, S E=0.15)$ and significantly different from zero $[t(18)=2.38]$. This result goes along with findings on RM effects in the literature, indicating that an observer's memory for a moving object (Hubbard, 1995 ) or for a photograph with implied motion (Freyd, 1983 ) is distorted in the direction of the suggested motion. This finding is also compatible with the hypothesis of an anticipation of the future posture in biological motion perception (Verfaillie \& Daems, 2002) and indicates that when a visual stimulation such as a PLC movement is abruptly stopped, its dynamics survive.

The ANOVA on PSE values showed that the average bias in the spatial layout condition was significantly greater $[F(1,18)=5.94]$ than in the condition without spatial layout. There was no significant main effect of conditions of observation on the average bias. However, the effect of conditions of observation on the memory bias varied as a function of spatial layout (see Figure 5), as indicated by the significant conditions of observation $\times$ layout interaction $[F(2,36)=10.62]$.

Our first hypothesis was that RM effects should diminish when an event is less predictable. Accordingly, we first examined memory bias for pan and track when 


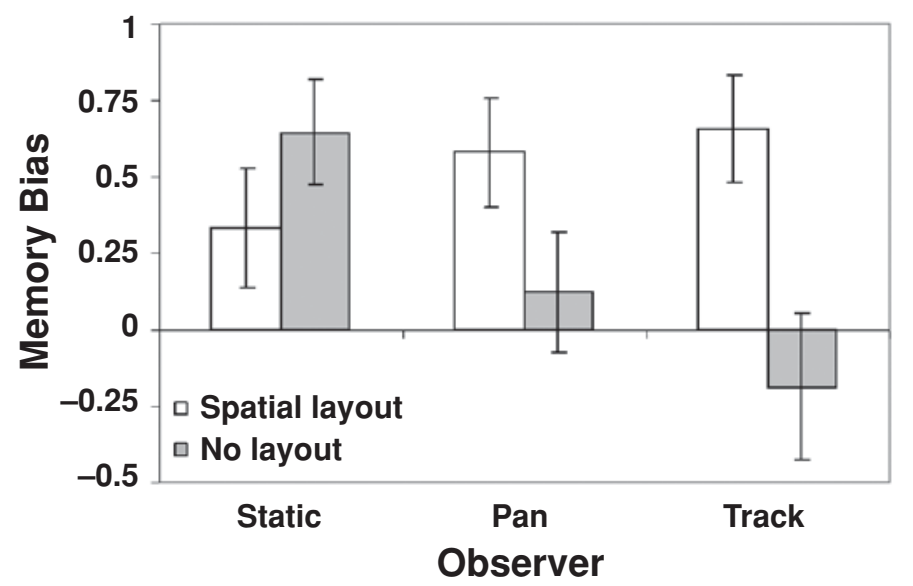

Figure 5. Observer $\times$ spatial layout interaction on average memory bias in Experiment 1.

the spatial layout induced an optic flow that informed the observer about the translational component of a roundoff/backward-somersault, as compared with the condition without spatial layout. With a Bonferroni correction $(p<.05 / 6 \approx p<.008)$, we found that the memory bias for pan and track with spatial layout differed significantly from zero $[t(18)=3.16$ and $t(18)=3.60$, respectively]. In contrast, without a layout that induced an optic flow, the memory bias for pan and track did not differ from zero. These results confirm that when there is no optic flow to specify the translational component of a PLC, the event is less predictable, and the anticipatory RM effect vanishes.

Among the questions addressed in Experiment 1 was the perceptual difference introduced by the pan and the track conditions. Although these two conditions are more or less equivalent in terms of velocity fields (Hochberg, 1986), the structure of the two flow patterns is clearly different, with a rotational component in the first case and a translational component in the second. In order to test whether the effect of global optic flow on memory bias depends on optic flow structure (pan vs. track), planned comparisons were performed. They revealed no difference in memory bias between the two conditions in the presence of a layout, reinforcing Hochberg's suggestion that different optic flow structures, such as pan and track shots, can be used to produce more or less the same flow of information about the whole scene. Finally, both conditions (pan and track) did not differ between each other in the absence of a layout.

Contrary to the pan and track conditions' involving mostly local rotational motion of the PLC (plus a small vertical component in the track condition), the static condition generates both a rotational motion of the PLC and a large horizontal linear component, since the PLC is translating across the screen during the round-off. Therefore, we expected that the static condition would provide enough visual information to initiate RM effects. With a Bonferroni correction $(p<.05 / 6 \approx p<$
$.008)$, the results showed that the memory bias in the static without spatial layout condition differed significantly from zero $[t(18)=3.61]$. However, in the presence of a spatial layout, the memory bias was not significantly different from zero, suggesting that the spatial layout provided informational cues for remembering the final posture accurately. This last result is consistent with Gray and Thornton's (2001) finding that RM effects are reduced when landmarks are used to disambiguate the point of disappearance of translating targets. Moreover, it is worth noting that, in the conditions producing an $\mathrm{RM}$ effect, although the visual stimulation was very different, the amplitude of the RM effect was roughly the same (95\% CI: static without layout $[+0.27,+1.02]$; pan with layout $[+0.19,+0.97]$; track with layout $[+0.27$, $+1.04]$ ), and the three conditions did not differ (according to Tukey's HSD post hoc test). Again, this suggests that when there is enough information available to extract the properties of an ongoing movement (e.g., the translational and rotational components of a complex biological motion), the movement becomes predictable and is subjected to RM effects when interrupted abruptly.

The ANOVA on the JND values of the memorized posture revealed that the imprecision observed in conditions without a spatial layout $(M=1.56, S E=0.09)$ was significantly greater $[F(1,18)=6.71]$ than with spatial layout $(M=1.35, S E=0.10)$. There was no significant main effect of conditions of observation on JND, but there was a significant conditions of observation $\times$ layout interaction $[F(2,36)=8.31]$. Post hoc HSD Tukey tests revealed that the track condition without a spatial layout led to larger JND values $(M=2.03, S E=0.14)$, which differed significantly from each of the other conditions and marginally from the static observer in the presence of a spatial layout $(p<.06)$. These results suggest that the track condition without a spatial layout led to a loss in precision of the remembered posture. The irregularity of the biological motion in this peculiar condition (see Figure 2) certainly affected the quality of the 
Table 1

Mean Performance $( \pm S E)$ for Each Dependent Variable as a Function of Angular Difference in Viewpoint, in Experiment 1

\begin{tabular}{|c|c|c|c|c|c|c|}
\hline \multirow[b]{2}{*}{ Viewpoint } & \multicolumn{2}{|c|}{$\begin{array}{c}\text { Memory } \\
\text { Bias }\end{array}$} & \multicolumn{2}{|c|}{ JND } & \multicolumn{2}{|c|}{$\begin{array}{c}\text { Reaction Time } \\
(\mathrm{msec})\end{array}$} \\
\hline & $M$ & $S E$ & $M$ & $S E$ & $M$ & $S E$ \\
\hline $0^{\circ}$ & 0.42 & 0.16 & 0.79 & 0.12 & 896 & 43 \\
\hline $30^{\circ}$ & 0.47 & 0.19 & 1.10 & 0.11 & 966 & 60 \\
\hline $60^{\circ}$ & 0.42 & 0.26 & 1.62 & 0.19 & 1,074 & 89 \\
\hline $90^{\circ}$ & -0.27 & 0.21 & 2.64 & 0.12 & 1,201 & 115 \\
\hline
\end{tabular}

Note-JND, just noticeable difference.

memorized posture (in terms of precision, not accuracy), because of the absence of movement information gathered by the visual system. Accordingly, in the absence of a spatial layout to specify the origin of the vertical component in the track condition (Figure 2), the motion was less predictable, and therefore, memorization performance was less precise.

The ANOVA on the RTs to the test posture showed no main effect for layout, no main effect for conditions of observation, and no interaction.

Viewpoint change. Interactions between angular difference in viewpoint and other experimental factors for PSE or JND could not be computed, due to an insufficient number of values per cell. Therefore, the results for the effect of viewpoint change will be presented separately from those for the other experimental factors. Note that for the purpose of data analysis, angular differences were grouped according to their absolute values $\left(0^{\circ}, 30^{\circ}\right.$, $60^{\circ}$, or $90^{\circ}$ ).

The ANOVA showed a significant effect of angular difference on PSE $[F(3,54)=3.34]$. This effect seems to have been due to the $90^{\circ}$ condition's leading to a much lower bias, as compared with the other conditions. Post hoc HSD Tukey tests revealed that the $90^{\circ}$ condition differed significantly from the $30^{\circ}$ condition and marginally from the $0^{\circ}(p<.07)$ and $60^{\circ}(p<.07)$ conditions, whereas the other conditions did not differ from each other. The fact that the viewpoint change coincided with the change from a tilted posture (at initial viewpoint $=$ $\left.0^{\circ}\right)$ to a more and more slanted posture may account for this result (see Figure 1A).

Figure 1A shows the shift from a tilted test posture to a slanted test posture when the angular difference in viewpoint increases. Considering that the environmental vertical can serve as a reference for specifying an orientation in tilt, but not in slant, it is not surprising that the $90^{\circ}$ viewpoint change affects perceptual judgments more than do the other viewpoints.

The ANOVA performed on JND values revealed a significant increase in JND $[F(3,54)=40.80]$ with the angular difference in viewpoint (see Table 1). Thus, the precision of the remembered posture decreased with an increasing angular difference in viewpoint. This result is consistent with the conclusions of several authors (Koenderink, van Doorn, \& Kappers, 1992; Oomes \& Dijkstra,
2002; Stevens, 1983), indicating that precision for orientations in the frontoparallel plane (tilt) is consistently higher (less variable responses) than that for orientations in depth (slant).

Finally, there was a significant increase in $\mathrm{RT}[F(3,54)=$ 9.41] with angular difference in viewpoint (see Table 1), in conformity with the results of Diwadkar and McNamara (1997), indicating that RTs increase linearly as a function of the angular distance between test and trained views.

\section{EXPERIMENT 2}

In Experiment 1, we showed that in the absence of optic flow specifying the translational component of the PLC movement, an event was less predictable and anticipatory RM effects did not occur. In Experiment 2, participants judged whether the same biological motion produced by the PLC was continuous despite viewpoint changes. We expected the amplitude of viewpoint change, not the initial observation condition, to affect performance, due to the availability of the biological motion input from the new viewpoint.

\section{Method}

Participants. Twenty-two new individuals, between 23 and 41 years of age, took part in Experiment 2. All had normal or correctedto-normal vision and were naive with respect to the hypothesis under investigation.

Apparatus and Stimuli. We used the same setup and stimuli as those in Experiment 1.

Procedure. In contrast with Experiment 1, the PLC did not disappear during the backward somersault. The PLC movement continued until its end, despite a possible viewpoint change, as in a cinematographic camera cut. The participants were instructed to observe the movement of the PLC and to indicate, after a possible change in the viewpoint, whether the PLC movement had been shifted backward or forward at the time of viewpoint change. The test posture was the first posture of the PLC seen from the new viewpoint. The different test postures were frames $n-2, n-1$, $n, n+1, n+2, n+3$, and $n+4$. Given that the last frame of the posture under the initial viewpoint was frame $n$, the correct test posture from the new viewpoint should be frame $n+1$. Responses were recorded via a keyboard (backward = left Ctrl key; forward = right Ctrl key). Each participant performed 294 experimental trials: 2 layout conditions (with or without a spatial layout) $\times 3$ conditions of observation (static vs. pan vs. track) $\times 7$ angular differences $\left(-90^{\circ},-60^{\circ},-30^{\circ}, 0^{\circ},+30^{\circ},+60^{\circ}\right.$, or $\left.+90^{\circ}\right) \times 7$ test postures $(n-2$ to $n+4)$. Lastly, the final posture from the initial

Table 2

Mean Performance $( \pm \mathrm{SE})$ for Each Dependent Variable as a Function of Angular Difference in Viewpoint, in Experiment 2

\begin{tabular}{|c|c|c|c|c|c|c|}
\hline \multirow[b]{2}{*}{ Viewpoint } & \multicolumn{2}{|c|}{$\begin{array}{c}\text { Perceptual } \\
\text { Bias }\end{array}$} & \multicolumn{2}{|c|}{ JND } & \multicolumn{2}{|c|}{$\begin{array}{l}\text { Reaction Time } \\
\quad(\mathrm{msec})\end{array}$} \\
\hline & $M$ & $S E$ & $M$ & $S E$ & $M$ & $S E$ \\
\hline $0^{\circ}$ & -0.28 & 0.14 & 0.39 & 0.09 & 828 & 50 \\
\hline $30^{\circ}$ & -0.32 & 0.16 & 1.16 & 0.18 & 890 & 42 \\
\hline $60^{\circ}$ & -0.94 & 0.20 & 1.42 & 0.16 & 901 & 43 \\
\hline $90^{\circ}$ & -2.56 & 0.48 & 2.66 & 0.33 & 973 & 48 \\
\hline
\end{tabular}

Note-JND, just noticeable difference. 
Left-Ankle-Head Axis Orientation (Frames 51 to 63)
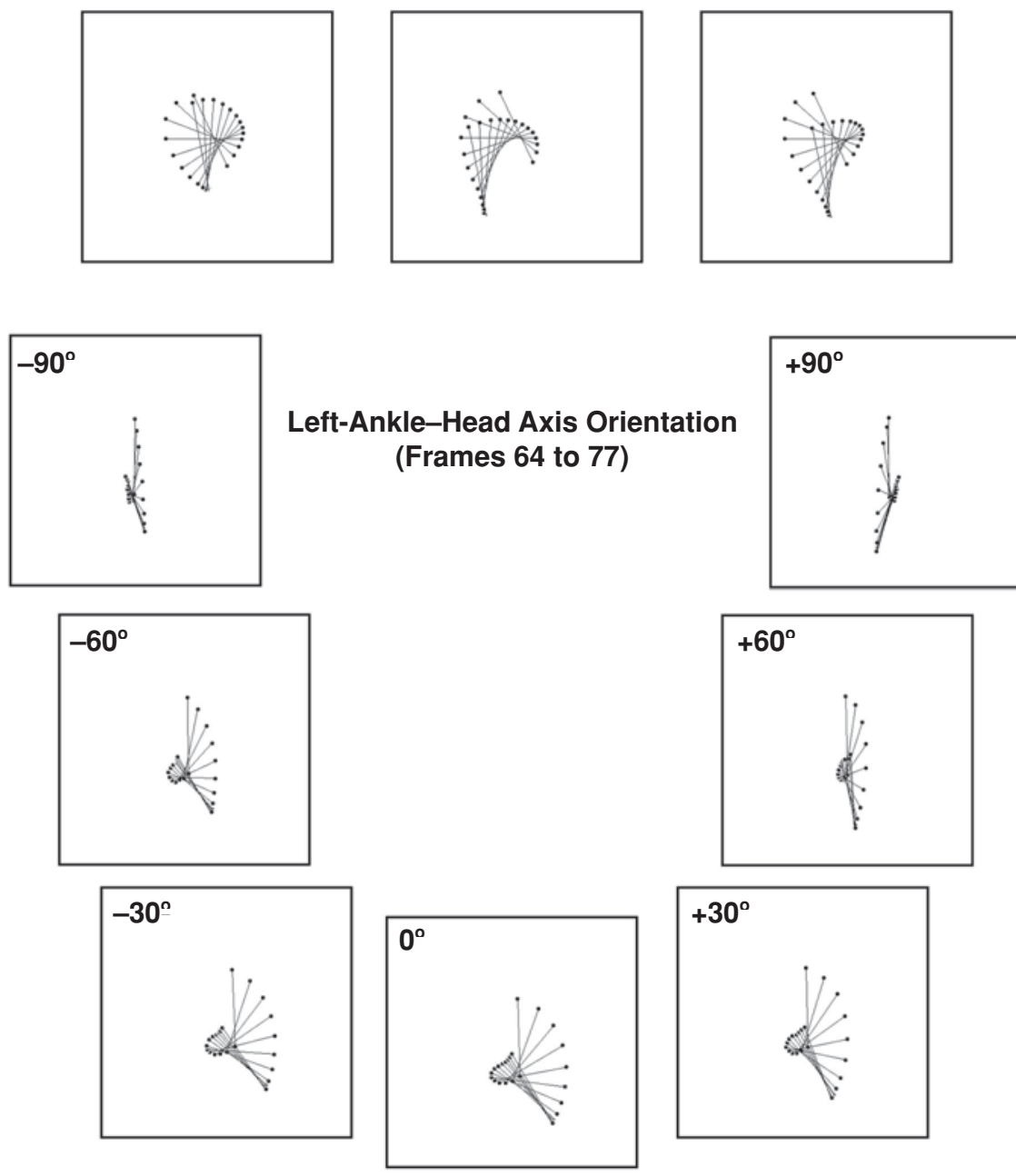

Figure 6. The top panel illustrates the trajectories of the head and ankle during the phase of lift off (from Frame 51 to Frame 63) of the somersault in the three conditions of observation (static, pan, and track). In the bottom panel, these trajectories are illustrated during the landing phase (from Frame 64 to Frame 77) of the somersault in the static condition and from the seven viewpoints $\left(-90^{\circ},-60^{\circ},-30^{\circ}, 0^{\circ},+30^{\circ},+60^{\circ}\right.$, and $\left.+90^{\circ}\right)$.

viewpoint could be that of Frame 63, 66, or 69. These final postures were crossed with the other factors in an incomplete balanced design (Cochran \& Cox, 1957).

Dependent variables. It has to be remembered that the participants were asked to detect the (dis)continuity in the PLC movement after a possible change in viewpoint. PSE values were computed from the responses to various frames, in order to infer the frame expected by the observer after the change in viewpoint. PSE corresponds to the perceptual bias reflecting the accuracy of the responses. Given that the final posture from the initial viewpoint corresponds to frame $n$, a PSE equal to 0 signifies that the participants accurately identified that, from the new viewpoint, the PLC movement restarted at frame $n+1$. A PSE equal to 1 signifies that the participants would have perceived the PLC movement as continuous if it had started at frame $n+2$ from the new viewpoint. Finally, a PSE equal to -1 indicates that the participants would have perceived the PLC movement as continuous if it had started at frame $n$ from the new viewpoint - that is, by replaying the final posture of the initial viewpoint (frame $n$ ).

JND values were computed to measure the precision of the perceived (dis)continuity - that is, the JND in motion continuity. RTs (in milliseconds) to the camera cut were also recorded, as in Experiment 1 .

\section{Results and Discussion}

In contrast to Experiment 1, the average bias was negative $(M=-0.88, S E=0.19)$ and was significantly different from zero $[t(21)=4.59]$. This may have been due to the task used in Experiment 2 (detection of motion continuity), which does not require high-level memory processes that are affected by RM effects (exhibiting positive bias values). An ANOVA showed a main effect of angular difference on perceptual bias $[F(3,63)=22.82]$. More precisely (see Table 2), with a Bonferroni correction $(p<.05 / 4 \approx p<.012)$, the mean bias for $0^{\circ}$ and $30^{\circ}$ did not differ significantly from zero, whereas the mean bias for $60^{\circ}$ and $90^{\circ}$ did $[t(21)=4.70$ and $t(21)=5.21$, respectively]. In other words, the perceptual bias was (negatively) smaller for high angular differences in view- 
point than for small angular differences. This may have been due to the amplitude of the PLC rotation during the somersault. If slant rotations are underestimated, for equivalent perceptual judgment of $30^{\circ}$ rotation, we would judge a slant rotation to be smaller in amplitude than an equivalent tilt rotation (see Figure 6). Accordingly, the angular difference effect would be due to the change from a tilt to a slant rotation percept. The more we deviate from $0^{\circ}$, the less precise the estimation of the PLC rotation.

An ANOVA on JND values and RTs was in concordance with the results on perceptual bias. For JND, the detection of motion discontinuity degraded rapidly with the increase in viewpoint change $[F(3,63)=35.29$; see Table 2 and Figure 6]. RTs increased significantly $[F(3,63)=$ 7.95] with angular difference in viewpoint (see Table 2 ). In other words, from a $90^{\circ}$ viewpoint, the observers would need to gather more information on the PLC rotation by replaying the posture motion about 2.5 frames back in time, in order to perceive an amount of (slant) rotation equivalent to that perceived had no viewpoint change occurred - that is, a tilt rotation from a viewpoint of $0^{\circ}$ (see Figure 6).

Regarding the effect of observation conditions and spatial layout, we expected that the structural and transformational invariants specifying the PLC movement would be sufficient for judgments of movement continuity across viewpoint changes. We therefore did not expect these conditions to affect visual performance. An ANOVA performed on PSE confirmed this hypothesis. There was no significant effect on average bias of spatial layout or conditions of observation. There was, however, a significant conditions of observation $\times$ spatial layout interaction $[F(2,42)=4.75]$. Post hoc HSD Tukey tests revealed that this interaction was due to a significantly more negative bias in the static condition than in the pan condition in the presence of a spatial layout. This result may have been due to the fact that in the pan condition with a spatial layout, because the event is specified in the optical flow, attention can be focused on the PLC mo- tion. There is, in contrast, no global flow in the static condition, but a static layout. In this condition, an observer may encode the posture relatively to the layout from both viewpoints, thereby paying less attention to motion continuity itself.

Consistent with PSE values, JND values were significantly $[F(1,21)=4.92]$ greater (i.e., the motion continuity judgments were less precise) in the presence of a spatial layout $(M=1.65, S E=0.17)$ than without a spatial layout $(M=1.43, S E=0.18)$. In addition, there was a significant main effect of conditions of observation on JND $[F(2,42)=3.24]$. Post hoc HSD Tukey tests revealed that JND values were significantly greater in the track condition $(M=1.72, S E=0.21)$ than in the static condition $(M=1.37, S E=0.18)$. This last finding nicely fits with the results of Experiment 1 showing that the more predictable the biological motion, the more precise the performance. There was no significant conditions of observation $\times$ spatial layout interaction on JND. The JND values in each condition are detailed in Figure 7.

We also tested, as in Experiment 1, whether the effect of global optic flow on perceptual bias depended on the structure of the optic flow (pan vs. track). Planned comparisons were performed that showed no difference in perceptual bias between the pan and the track conditions when the spatial layout was present (see Figure 7). This result is again consistent with the results of the first experiment, supporting Hochberg's (1986) assertion that filmmakers can use various optical flow structures, such as pan and track shots, in order to produce equivalent perceptual effects.

Finally, an ANOVA on the RT to the motion (dis)continuity indicated no significant main effect for spatial layout or conditions of observation. Although these two factors interacted $[F(2,42)=4.64]$, post hoc HSD Tukey tests revealed that none of the pairwise comparisons reached significance. As in Experiment 1, interactions between angular differences in viewpoint and other experimental factors on memory bias or JND could not be computed, because of an insufficient number of values per cell.

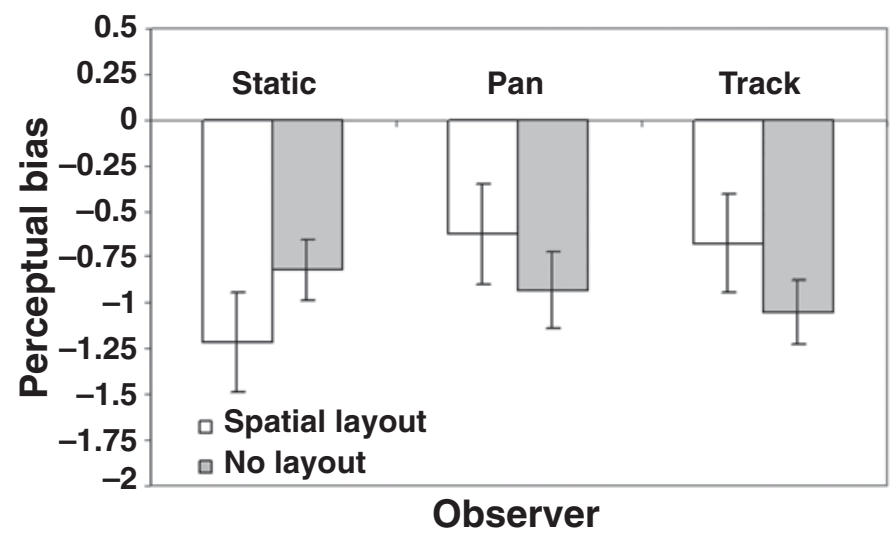

Figure 7. Observer $\times$ spatial layout interaction for average perceptual bias in Experiment 2. 


\section{GENERAL DISCUSSION}

In this study, the effects of visual self-motion and viewpoint changes on the perception and memorization of biological motion were examined. In a first experiment, participants memorized the final posture of an interrupted biological motion (round-off/backward-somersault), in order to decide whether a subsequent test posture was located after or before the actual final posture. In a second experiment, participants judged whether the biological motion appeared to be continuous across a viewpoint change. Two main results can be summarized from the present study. First, for ambiguous conditions of observation, such as pan and track, in the absence of optic flow specifying the translational component (no layout conditions), the event was less predictable, and RM effects were reduced (Experiment 1). Second, the structural and transformational invariants specifying the movement were sufficient to judge whether the motion was continuous across a viewpoint change. In this case, it was the amplitude of the viewpoint change, rather than the conditions of observation, that modulated the perceived event continuity (Experiment 2). These findings support the adaptive value of predictability of our visual system (see Thornton \& Hubbard, 2002).

In the first experiment, we found that when the pan and track conditions induced an optical flow that was informative about the perceived event, RM effects occurred. This result suggests (at least in the conditions under investigation) that it is more the presence of global optical flow than its structure (translational or rotational) that modulates the perception of PLC movement, since we found no difference in the flow-induced RM effect between the pan and the track conditions. These results are consistent with filmmakers' empirical findings that various techniques (pan or track) can be used to simulate similar flows of information about a whole scene (Hochberg, 1986). Our data are complementary to those in Whitney and Cavanagh (2002) and in Thornton and Hayes (2004), showing that RM is affected by surrounding motion and self-motion. They also indicate that when the observer is static, the spatial layout provides potential spatial references for remembering the final posture accurately, thereby diminishing the RM effect. The last result generalizes, to more complex cultural movements, Gray and Thornton's (2001) finding that, for a translating target, when landmarks are used to disambiguate the point of disappearance, RM is reduced.

In Experiment 2, when the information specifying the event was continuously available in the PLC movement, the initial conditions of observation did not contribute to the detection of motion continuity. However, the greater the amplitude of viewpoint change, the more difficult the detection of motion discontinuity across viewpoint change. In this experiment (as well as in Experiment 1), perception and memorization of PLC movement were affected for large angles $\left(60^{\circ}\right.$ and $\left.90^{\circ}\right)$. What can account for this effect?
A first explanation is related to the amplitude of PLC rotation during the somersault. As was stated in the Results section for Experiment 2, for an equivalent amplitude of rotation of the PLC, observers would judge slant rotations as being of smaller amplitude than were equivalent tilt rotations (see Figure 6), introducing imprecision and underestimation in perceptual judgments (see, e.g., Koenderink et al., 1992; Oomes \& Dijkstra, 2002).

Alternatively, this effect may be the consequence of perceptual learning. An illustration of the perceptuallearning account of viewpoint effects has been provided by Stephenson and Jackson (1977), exploring the effects of training position on judges' ratings at a gymnastics event. They found evidence that judges were able to detect a greater number of execution faults when they observed the performance from the front view, rather than from the side view, because judges are usually trained from front views - that is, with slant perspective. In contrast to the participants in Stephenson and Jackson, who had problems with tilt angles, our participants had difficulties with slant angles. An explanation could be that our observers were initially familiarized with PLC movements from the $0^{\circ}$ viewpoint. In other words, they were perceptually trained with tilt rotations of the PLC during its movement. Moreover, they performed the experimental trials while adopting an initial viewpoint at $0^{\circ}$, corresponding to a tilt rotation of the PLC. As a consequence, the round-off was always perceived as a tilt rotation, and the backward somersault could shift from a tilt to a slant rotation, depending on the amplitude of the viewpoint change (see Figures 2 and 6). As a consequence, in our study, visual expertise at tilt interfered with judgments of slanted orientations.

Finally, a third alternative account of the effect of viewpoint change on the perception of continuity would be related to change blindness - that is, the inability to detect changes from one view of a scene to the next (Simons \& Levin, 1997). It is a well-documented fact that when cutting on motion (i.e., performing a camera cut while observing a moving character or an object), change blindness occurs (Hochberg, 1986; Simons \& Levin, 1997). There are several classical examples of change blindness, such as the unnoticed change of the location of a jacket (from the shoulders of a sitting person in a cafeteria to the back of his/her chair) that accompanies a camera cut (e.g., Rensink, 2002; Simons, 2000) or the replacement after the cut of the main actor by another actor completing the action (Simons \& Levin, 1997). One may assume that change blindness would increasingly degrade motion continuity judgments with greater amplitudes in viewpoint change. Somehow, the binding of motion across camera cut would be more difficult with an increasing amplitude in viewpoint change.

In many experimental studies, simple object motion has been used to show the effect of a surrounding context on memory shift (Thornton, 2002; Whitney \& Cavanagh, 2002) or perceptual motion continuity across camera cut (e.g., Hochberg, 1986; Simons \& Levin, 1997). In our 
study, we used realistic biological motion to show the effects of global optical flow on the perceiving and remembering of a complex motion stimulus. The findings reported in these two experiments have important implications for the perceiving and judging of complex biological motions. We are convinced that our results are not specific to biological motion per se, but to event perception in general, and that they could be extended to simpler, two-dimensional or 3-D object motion, provided that the movement of the object has linear and rotational components. Biological motions are not immune to memory and perceptual biases from RM effects and observation conditions. This line of reasoning may help us to understand judging errors during the perception of actions and postures in sports, a research direction that is currently under investigation.

\section{REFERENCES}

Abernethy, B. (1987). Selective attention in fast ball sports: II. Expertnovice differences. Australian Journal of Science \& Medicine in Sport, 19, 7-16.

Alain, C., \& Proteau, L. (1980). Decision making in sport. In C. H. Nadeau, W. R. Halliwell, K. M. Newell, \& G. C. Roberts (Eds.), Psychology of motor behavior and sport (pp. 465-477). Champaign, IL: Human Kinetics.

ARIJon, D. (1993). Grammaire du langage filmé: Encyclopédie de la mise en scène (4th ed.). Paris: Editions Dujarric.

BERINGER, J. (1994). ERTS-IPL: Tachistoscopic color image displays and accurate response registration on IBM PCs [Abstract]. Psychology Software News, 5, 37-38.

Cochran, W. G., \& Cox, G. M. (1957). Experimental designs. Toronto: Wiley.

DitTRICH, W. H. (1993). Action categories and the perception of biological motion. Perception, 22, 15-22.

DiWADKaR, V. A., \& MCNamara, T. P. (1997). Viewpoint dependence in scene recognition. Psychological Science, 8, 302-307.

D'Ydewalle, G., \& Vanderbeeken, M. (1990). Perceptual and cognitive processing of editing rules in film. In R. Groner, G. d'Ydewalle, $\&$ R. Parham (Eds.), Studies in visual information processing: Vol 1. From eye to mind: Information acquisition in perception, search, and reading (pp. 129-139). Amsterdam: Elsevier, North-Holland.

Finke, R. A., Freyd, J. J., \& Shyi, G. C. (1986). Implied velocity and acceleration induce transformations of visual memory. Journal of Experimental Psychology: General, 115, 175-188.

FREYD, J. J. (1983). The mental representation of movement when static stimuli are viewed. Perception \& Psychophysics, 33, 575-581.

FreYD, J. J. (1987). Dynamic mental representations. Psychological Review, 94, 427-438.

Gibson, J. J. (1950). Perception of the visual world. Boston: Houghton Mifflin.

GiBSON, J. J. (1958). Visually controlled locomotion and visual orientation in animals. British Journal of Psychology, 49, 182-194.

Gray, R., \& THORNTON, I. M. (2001). Exploring the link between time to collision and representational momentum. Perception, 30, 1007-1022.

HOCHBERG, J. (1986). Representation of motion and space in video and cinematic displays. In K. R. Boff, L. Kaufman, \& J. P. Thomas (Eds.), Handbook of perception and human performance: Vol. 1. Sensory processes and perception (pp. 22.1-22.64). New York: Wiley.

HUBbard, T. L. (1995). Environmental invariants in the representation of motion: Implied dynamics and representational momentum, gravity, friction, and centripetal force. Psychonomic Bulletin \& Review, 2, 322-338.

HubBard, T. L., \& Bharucha, J. J. (1988). Judged displacement in apparent vertical and horizontal motion. Perception \& Psychophysics, 44, 211-221.

JohANSSON, G. (1973). Visual perception of biological motion and a model for its analysis. Perception \& Psychophysics, 14, 201-211.
Koenderink, J. J., van Doorn, A. J., \& Kappers, A. M. L. (1992). Surface perception in pictures. Perception \& Psychophysics, 52, 487-496.

Michaels, C. F., \& Carello, C. (1981). Direct perception. Englewood Cliffs, NJ: Prentice Hall.

Oomes, A. H. J., \& Dijkstra, T. M. H. (2002). Object pose: Perceiving 3-D shape as sticks and slabs. Perception \& Psychophysics, 64, 507520.

Rensink, R. A. (2002). Change detection. Annual Review of Psychology, 53, 245-277.

Runeson, S., \& Frykholm, G. (1983). Kinematic specification of dynamics as an informational basis for person-and-action perception: Expectation, gender recognition, and deceptive intention. Journal of Experimental Psychology: General, 112, 585-615.

Shiffrar, M., \& Freyd, J. J. (1990). Apparent motion of the human body. Psychological Science, 1, 257-264.

Simons, D. J. (2000). Current approaches to change blindness. Visual Cognition, 7, 1-15.

Simons, D. J., \& LeVIN, D. T. (1997). Change blindness. Trends in Cognitive Sciences, 1, 261-267.

STE-Marie, D. M. (2000). Expertise in women's gymnastic judging: An observational approach. Perceptual \& Motor Skills, 90, 543-546.

Stephenson, D. A., \& JACKson, A. S. (1977). The effects of training and position on judges' ratings of a gymnastic event. Research Quarterly, 48, 177-180.

Stevens, K. A. (1983). Slant-tilt: The visual encoding of surface orientation. Biological Cybernetics, 46, 183-195.

Thornton, I. M. (2002). Further explorations of the onset repulsion effect. In M. Baumann, A. Keinath, \& J. F. Krems (Eds.), Experimentelle Psychologie: Abstracts der 44. Tagung experimentell arbeitender Psychologen. Regensburg, Germany: S. Roederer.

Thornton, I. M., \& Hayes, A. E. (2004). Anticipating action in complex scenes. Visual Cognition, 11, 341-370.

ThORNTON, I. M., \& Hubbard, T. L. (EDS.) (2002). Representational momentum: New findings, new directions. Hove, U.K.: Psychology Press.

Thornton, I. M., Pinto, J., \& Shiffrar, M. (1998). The visual perception of human locomotion. Cognitive Neuropsychology, 15, 535-552.

Verfaillie, K. (2000). Perceiving human locomotion: Priming effects in direction discrimination. Brain \& Cognition, 44, 192-213.

Verfaillie, K., \& Daems, A. (2002). Representing and anticipating human actions in vision. Visual Cognition, 9, 217-232.

WARREN, W. H., JR. (1995). Self-motion: Visual perception and visual control. In W. Epstein \& S. J. Rogers (Eds.), Handbook of perception and cognition: Perception of space and motion (2nd ed., pp. 263325). San Diego: Academic Press.

Warren, W. H., Jr., Mestre, D. R., Blackwell, A. W., \& Morris, M. W. (1991). Perception of circular heading from optical flow. Journal of Experimental Psychology: Human Perception \& Performance, 17, 28-43.

Whitney, D., \& Cavanagh, P. (2002). Surrounding motion affects the perceived locations of moving stimuli. Visual Cognition, 9, 139-152.

Williams, A. M., Davids, K., Burwitz, L., \& Williams, J. G. (1994). Visual search strategies of experienced and inexperienced soccer players. Research Quarterly for Exercise \& Sport, 65, 127-135.

Yela, M. (1952). Phenomenal causation at a distance. Quarterly Journal of Experimental Psychology, 4, 139-154.

\section{NOTES}

1. Whereas optic flow (global or local) is unaffected by eye movements, retinal flow - that is, the sampling of the optical flow by a mobile eyeis influenced by the direction of glance and the rotation of the eye (see, e.g., Warren, 1995).

2. These flow patterns are those entering the eye of a virtual observer. In screen coordinates - that is, for a (static) external observer looking at the monitor screen - the PLC is translating and rotating across the screen in the static condition but is only rotating in pan and track conditions, providing additional retinal information.

(Manuscript received March 1, 2004; revision accepted for publication November 11, 2004.) s 